\title{
Simulation of coupled sputter-diffusion effects
}

\author{
U. von Toussaint ${ }^{1}$, A. Mutzke $^{1}$, K. Sugiyama ${ }^{1}$, T. Schwarz-Selinger ${ }^{1}$ \\ ${ }^{1}$ Max-Planck-Institut für Plasmaphysik, EURATOM Association, Boltzmannstr. 2, 85748 \\ Garching, Germany \\ E-mail: udo.v.toussaint@ipp.mpg.de
}

\begin{abstract}
The simultaneous effects of sputtering, implantation and solid-state diffusion determine the surface profiles of mixed-material systems under ion-bombardment at elevated temperatures due to the enhanced atomic mobility. To simulate the joint processes the Monte Carlo code SDTrimSP for the simulation of the ion-solid interaction has been augmented by a diffusion model for solid-state diffusion. The combined model has been applied to a tungsteniron system under deuterium bombardment as model system for EUROFER. The simulation results reveal a strong dependence of the surface profile on initial tungsten concentration, ion energy, temperature and fluence but also on the impinging flux, a parameter which is often not appropriately taken into account. For reactor relevant parameters of low-energy $(200 \mathrm{eV})$ deuterium fluxes of $10^{21} \mathrm{at} / \mathrm{m}^{2} \mathrm{~s}$ at $873 \mathrm{~K}$ a tungsten-iron system exhibits an increase of the tungsten surface concentration from initially $1 \%$ by a factor of more than 20 , which drops at lower fluxes.
\end{abstract}

PACS numbers: $52.40 . \mathrm{Hf}, 66.30 . \mathrm{J}-$, 89.30.Jj

Submitted to: Phys. Scr. / PFMC-15

\section{Introduction}

The plasma-facing wall of next step fusion devices should ensure long-time operation and needs to be compatible with stringent constraints on hydrogen isotope (i.e. tritium) retention and permeation. Tungsten is presently considered as one of the most favorable materials for plasma-facing components due to its low physical sputtering and its low solubility for hydrogen. However, under the $14 \mathrm{MeV}$ neutron irradiation conditions of such devices the microstructure of the material will change and result in increased embrittlement. Therefore the possibility to use radiation-resilient low-activation steels (e.g. EUROFER, typically containing $\leq 1 \mathrm{wt} . \% \mathrm{~W}$ ) as plasma-facing material on the main vessel wall appears tempting. Deuterium sputtering experiments on EUROFER and on Fe-W-model systems at PISCES A at $373 \mathrm{~K}$ revealed a progressively reduced sputtering yield of about one order of magnitude with deuterium fluence 1 due to surface enrichment of tungsten for deuterium energies of $140 \mathrm{eV}$. The reduced sputter yield for iron was robust for a wide temperature range up to $773 \mathrm{~K}$. For temperatures above $800 \mathrm{~K}$ the sputter yield increased strongly [2]. It was conjectured that the surface enrichment is limited at elevated temperatures (i.e. in the range of targeted first-wall 


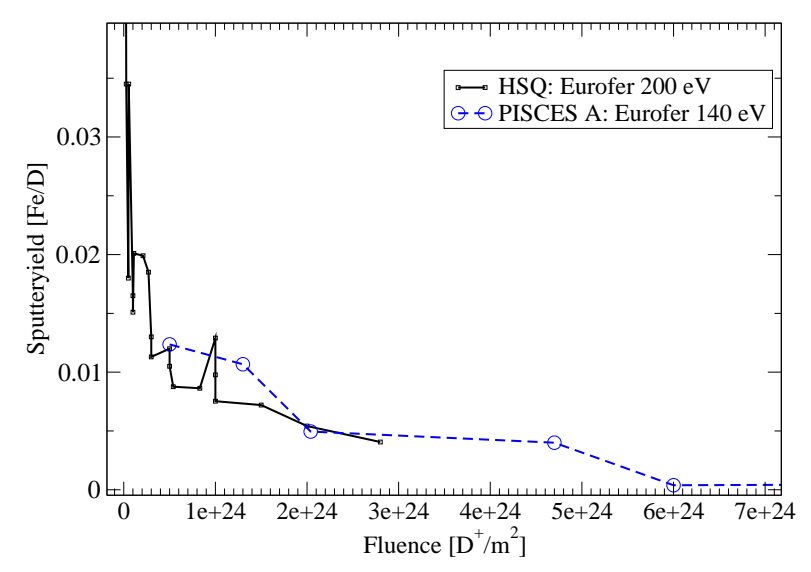

Figure 1. Fluence dependence of the sputtering yield of EUROFER under exposure of deuterium plasma in PISCES A with a bias of $-150 \mathrm{~V}$ (open symbols). For comparison the measured erosion yield dependency for EUROFER under a mass-separated, monoenergetic $200 \mathrm{eV}$ D-ion beam is shown (solid line). In both cases a significant drop of the erosion yield of iron with fluence is observed. Experimental details are given in [1].

operation temperatures) by the onset of solid-state diffusion. To take diffusion effects into account we enhanced the Monte Carlo code SDTrimSP [3] for ion-solid-interaction with the capability to compute solid-state diffusion based on Fick's laws. This allows to consider the coupled effects of implantation, sputtering and diffusion in an integrated way.

\section{The Modeling Approach}

There have been previous attempts to incorporate the influence of diffusion on ion-solid interactions, see e.g. [4, 5], by copying concentration profiles back and forth between codes modeling the diffusion and codes modeling the collision cascades. Unfortunately, the coordination of independent programs proved to be cumbersome and of limited efficiency. However, a closer look at the internal structure of the code SDTrimSP revealed that most of the prerequisites for the modeling of solid-state diffusion were already in place. The basic loop of SDTrimSP which is iterated until the desired projectile fluence consists of the following steps

- Impact of the projectile (actually a metaparticle)

- Computation of initial projectile-particle collision and the subsequent collision-cascades of both, projectile and recoils

- Registration of the final positions of all involved particles

- Update of the sample structure due to the computed collision cascades

The life-time of ion-induced collision cascades is of the order of picoseconds, which is many orders of magnitude smaller than relevant time scales for solid-state diffusion. Due to this scale-separation it is possible to consider in the basic loop of SDTrimSP also additional 
diffusion processes, logically changing the last step to 'Update of the sample structure due to the computed collision cascades and diffusion'.

\subsection{The diffusion constant}

In contrast to the binary systems of hydrogen in metals where the definition of the diffusion constant of hydrogen is obvious and undisputed, in solid-state diffusion several diffusion constants are routinely used [6,7], which apply to very specific situations, i.e. specific choices of reference systems with often subtle differences and implicit assumptions (cf. constant density [8]). Since we need the species resolved local mobility of the atoms the present implementation in SDTrimSP uses the intrinsic diffusion or component diffusion coefficient $D$, which describes the diffusion of the individual species in a local and fixed coordinate system [7]. However, the typically measured values in an interdiffusion experiment refer to a different choice of the coordinate system: Since the diffusion rates of A or B atoms of a binary A-B alloy are usually not equal, in interdiffusion experiments a net flux of atoms across any lattice plane exists, resulting in the Kirkendall effect [9] and the corresponding Kirkendall velocity measured in a global reference system. However, these experimentally determined interdiffusion or chemical diffusion coefficients $\tilde{D}$ can be converted to the required intrinsic diffusion coefficients $D$ via the Manning relations [10].

The temperature dependence of the diffusion coefficients is assumed to be described by an Arrhenius relation

$$
D=D_{0} \exp \left(\frac{\Delta H}{k_{B} T}\right),
$$

with $D_{0}$ denoted as pre-exponential factor (unit: $\mathrm{m}^{2} / \mathrm{s}$ ), $\Delta H$ as activation enthalpy (unit: $\mathrm{eV}$ per atom), with Boltzmann constant $k_{B}$ and temperature $T$ (in Kelvin).

\subsection{Implemented Algorithms}

The implementation of the 1-d diffusion algorithm has to take into account the specifics of the code SDTrimSP: neighboring slabs may have different thicknesses $\Delta x_{j}$ and different densities, which we suppress in the following to avoid a too cluttered notation. In addition a temperature gradient may be present. Therefore locally varying diffusion coefficients have to be taken into account. We have implemented three different algorithms with different specifics for the computation of the diffusion.

\subsubsection{Explicit algorithm Differencing the diffusion equation}

$$
\frac{\partial c}{\partial t}=\frac{\partial}{\partial x}\left(D(x) \frac{\partial c}{\partial x}\right)
$$

yields the explicit forward-in time, centered in space (FTCS) equation [11] for the concentration $c_{j}^{n+1}$ in slab $j$ in time step $n+1$,

$$
\frac{c_{j}^{n+1}-c_{j}^{n}}{\Delta t}=\frac{D_{j+1 / 2}\left(c_{j+1}^{n}-c_{j}^{n}\right)-D_{j-1 / 2}\left(c_{j}^{n}-c_{j-1}^{n}\right)}{(\Delta x)^{2}}
$$


where $D_{j+1 / 2}$ denotes the diffusion constant at the interface between slab $j$ and $j+1$. This algorithm is stable for time steps $\Delta t$ with

$$
\Delta t \leq \min _{j}\left(\frac{(\Delta x)^{2}}{2 D_{j+1 / 2}}\right) .
$$

This is a severe restriction in some cases because slab-widths of the order of $10^{-9}-10^{-10} \mathrm{~m}$ are not uncommon, thus enforcing very small time steps. This disadvantage can be overcome with implicit methods.

2.2.2. Fully implicit algorithm An unconditionally stable algorithm has been implemented based on the backward in time equation

$$
\frac{c_{j}^{n+1}-c_{j}^{n}}{\Delta t}=\frac{D_{j+1 / 2}\left(c_{j+1}^{n+1}-c_{j}^{n+1}\right)-D_{j-1 / 2}\left(c_{j}^{n+1}-c_{j-1}^{n+1}\right)}{(\Delta x)^{2}},
$$

which appears very similar to Eq. 3, except that the spatial derivatives on the right hand side need to be evaluated at time-step $n+1$ and thus are unknown. To solve Eq.5 a tridiagonal system for the $c_{j}^{n+1}$ has to be solved at each time-step. This tridiagonal system can be solved with an effort scaling as in $O(N)$, with $N$ the number of slabs. However, this fully-implicit scheme is only first-order accurate in time [12], which can be improved by combining Eq.3 and Eq.5 appropriately into the Crank-Nicolson algorithm.

2.2.3. Crank-Nicolson algorithm The Crank-Nicolson algorithm is given by the average of the explicit and the implicit scheme, which provides a scheme which is centered at time-step $n+1 / 2$ for $f=1 / 2$ and thus second order in time [13]:

$$
\begin{aligned}
\frac{c_{j}^{n+1}-c_{j}^{n}}{\Delta t} & =f \frac{D_{j+1 / 2}\left(c_{j+1}^{n}-c_{j}^{n}\right)-D_{j-1 / 2}\left(c_{j}^{n}-c_{j-1}^{n}\right)}{(\Delta x)^{2}}+ \\
& +(1-f) \frac{D_{j+1 / 2}\left(c_{j+1}^{n+1}-c_{j}^{n+1}\right)-D_{j-1 / 2}\left(c_{j}^{n+1}-c_{j-1}^{n+1}\right)}{(\Delta x)^{2}} .
\end{aligned}
$$

The Crank-Nicolson method is often the suggested approach for diffusion problems (see e.g. [11]) due to its second order convergence but for the present application (see below) we recommend the fully implicit method instead.

\subsection{Code Verification and Validation}

The implemented diffusion codes have been verified against known analytic solutions of different diffusion problems, like spreading of delta-like layers, step functions and concentration profiles of Gaussian shape. A typical result is displayed in figure 2, where the time evolution of an initial step profile is computed with the three implemented algorithms (explicit, implicit and Crank-Nicolson) and compared with the analytic solution in the upper left panel. Only very minor differences can be seen after the first time step displayed and the results at later times are virtually identical. As a rule of thumb: for parameters where 
the maximum change of concentration was below 0.01 per cycle the code never caused any problems. It should be pointed out that the Crank-Nicolson algorithm may be prone (under extreme circumstances) to oscillatory behavior [14], i.e. if discontinuous initial conditions are present. Those oscillations are typically damped as time goes on and the solution becomes smooth. However, here we are modeling simultaneously the enrichment or depletion due to ion-solid interaction, which can act as a constant source of distortion and thus be detrimental to the properties of the Crank-Nicolson algorithm. Therefore we prefer the L-stable [15] fully implicit algorithm - because its main disadvantage (only first order convergence) does not play a role: The concentration profile is altered anyway by the collision cascade and thus there is an intrinsic limit on the length of the time steps and required accuracy in the diffusion step.
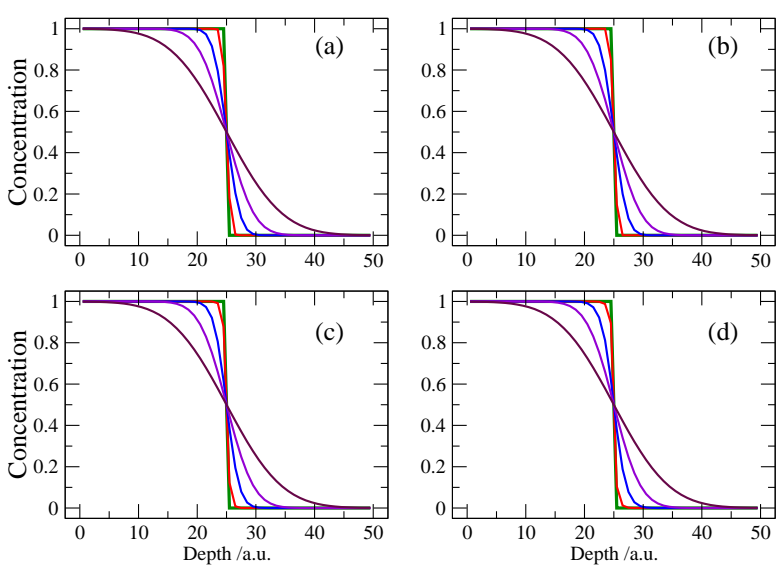

Figure 2. Time evolution of a concentration profile: The initial step function smears out and the maximum slope decreases monotonously. The analytic solution is given in subfigure (a), in (b) the result of the explicit algorithm is provided, in (c) the Crank-Nicolson results are displayed and (d) yields the data of the fully implicit algorithm. The differences on the short time evolution are hardly visible and the latter concentration profiles are virtually identical.

For further validation data available in literature, i.e. [4] on tungsten-carbon systems have successfully been reproduced.

\subsection{Further aspects}

In a large number of simulations of the tungsten-iron system the coupling of the Monte Carlo code and the diffusion module has turned out to be pretty robust. Here we want to mention parameter settings which lead to unexpected results and provide the rational for it. This may help to circumvent possible pitfalls and erroneous conclusions. Consider for example the homogeneous model system of 90 at.\% iron and 10 at.\% tungsten under $300 \mathrm{eV}$ deuterium ion bombardment at $473 \mathrm{~K}$ sample temperature. The sample is modeled by 1000 slabs, each having an initial width of $10 \AA$. For these parameters diffusion is almost negligible and the ion energy is above the sputtering threshold of both, iron and tungsten. Based on the difference in the sputter yield one would expect a stronger erosion of iron compared to tungsten and thus an increase of the tungsten surface concentration with fluence until an equilibrium concentration 
is reached. The simulation results are given in Fig. 3. Initially the tungsten concentration increases up to roughly 90 at. $\%$ at a deuterium fluence of $7.5 \times 10^{21}$ at $/ \mathrm{m}^{2}$. Then the tungsten concentration experiences several sharp drops, each followed by a smooth increase. Above a deuterium fluence of $1.5 \times 10^{22} \mathrm{at} / \mathrm{m}^{2}$ the concentration profile is again relatively smooth, but at the upper end of the fluence range a less pronounced oscillatory pattern in the concentration appears once more. Closer investigation reveals that the concentration fluctuations are caused by the slab merging algorithm of SDTrimSP: If a slab becomes to thin, it is combined with the neighboring layers. If the concentrations in these layers are too different then the merging process leads to concentration fluctuations. The onset and offset of the oscillations is determined by the composition dependent profile of forward implantation of iron and tungsten atoms which results in an iron enriched sub-implantation zone, resulting in an altered merging pattern of the slabs. Due to the influence of the forward implanted particles at later times (i.e. higher fluence) cycles with very long periods can be induced $[16,17]$.

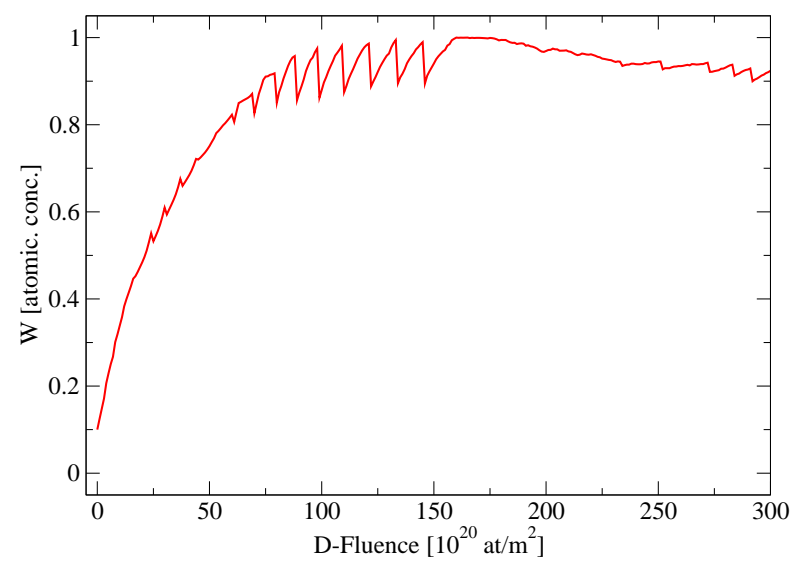

Figure 3. Surface concentration as function of deuterium fluence with $300 \mathrm{eV}$ and a flux of $10^{15} \mathrm{D} / \mathrm{m}^{2} \mathrm{~s}$. The initial sample composition was 90 at. $\% \mathrm{Fe}$ and 10 at.\% W. After the expected enrichment spurious sudden drops of the tungsten concentration are caused by merging of the increasingly eroded surface slab with the slab below if the initial discretization was too coarse.

An obvious solution to this undesired oscillatory behavior is the use of slab thicknesses such that the extend of the sputter-depleted zone is large compared to it. This ensures similar concentration profiles in neighboring layers, thus suppressing the merger induced fluctuations in the concentration profile at the expense of a largely increased computational effort. However, for some conditions (i.e. low ion-energy) the resolution to resolve the depletion profile needs to be better than $1 \AA$. An example for such a high-resolution simulation with slab widths of $0.2 \AA$ is provided in Fig. 4. The depth profile of the iron concentration displays the depletion of iron (and the corresponding enrichment of tungsten) by the ionbombardment at the surface, followed by a sharp increase in the iron concentration beyond $1 \AA$ reaching concentration values above the initial concentration at depths larger than $2 \AA$ due to recoil implantation. However, the peak of the iron concentration at the very surface appears suspicious. The peak results from the applied surface binding energy: Sputtered atoms even with a very low energy (few eV) are capable of penetrating several slabs because of the low 


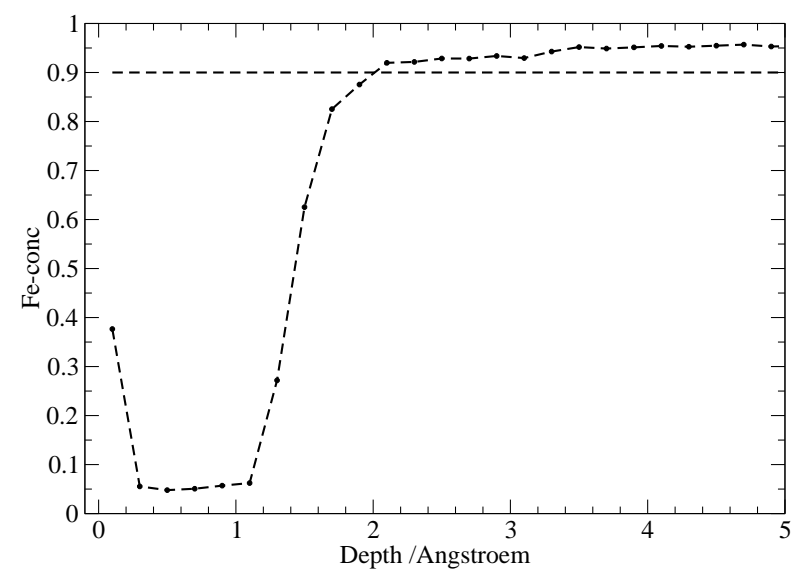

Figure 4. The high-resolution iron concentration profile after a fluence of $3 \times 10^{22} \mathrm{D} / \mathrm{m}^{2}$ $200 \mathrm{eV}$ deuterium atoms at $473 \mathrm{~K}$ sample temperature at a flux of $10^{15} \mathrm{D} / \mathrm{m}^{2} \mathrm{~s}$. The initial sample composition was 90 at.\% Fe and 10 at.\% W. The preferential sputtering by the ionbombardment results in a shallow iron depletion at the surface. The surface binding energy results in a increased iron concentration in the uppermost slab.

collision probability in each slab and are finally trapped in the outermost layer if the kinetic energy of the atom is below the surface binding energy. This appears unrealistic. In addition, slab widths below the lattice constant stretch the underlying physics model. However, the concept of surface binding energy is essential for binary collision approximation based codes working with repulsive potentials like SDTrimSP [3] or SRIM [18] and cannot simply be abandoned. Therefore, if sub-Angstrom slab widths are really required we recommend to exclude the uppermost layer in reporting the surface enrichment. The amplitude of the spurious surface peak decreases with increasing slab thickness and becomes negligible for slab thicknesses above approximately one atomic layer. Please note that the mentioned effects are not related to the new diffusion model but that they may be especially exposed by the parameter settings required for the present study.

\section{Properties of the iron-tungsten model system and simulation results}

The results of [1] of reduced sputter yields of EUROFER with increasing $140 \mathrm{eV}$ deuterium fluence and Rutherford backscattering analysis indicate an surface enrichment of tungsten in a very shallow surface layer. For controlled investigations of the enrichment process and comparison of experiment and simulation a binary model system iron-tungsten was selected. This avoids complications due to the complex chemical and morphological structure of steels.

For sufficiently large iron concentrations the tungsten-iron system is presumably a substitutional alloy. This favors a vacancy-based diffusion mechanism [6]. The estimated activation enthalpies for the interdiffusion in the iron-tungsten system exhibit some scatter, ranging from $2.22 \mathrm{eV}$ [19] to $2.77 \mathrm{eV}$ [20] to $2.97 \mathrm{eV}$ [21], which have to be related to the activation enthalpy of the self-diffusion of tungsten of $5.45 \mathrm{eV}$ and of iron $(2.80 \mathrm{eV})$. The activation enthalpies of the binary iron-tungsten system are similar or slightly below 
the values measured in austenitic steels with tungsten as component [22], ranging from 2.77 $\mathrm{eV}$ to $3.21 \mathrm{eV}$. In none of the diffusion studies evidence for the presence of a concentration dependent chemical factor [7] (influencing the diffusion, $D=D(c)$ ) has been found, allowing to simplify Manning's equation [7]. Therefore we choose the data of [21] for our iron component diffusion coefficient. The high activation enthalpy of tungsten would suppress any relevant diffusion of tungsten $\left(D_{\mathrm{W}} \approx 0\right)$. However, under ion-beam bombardment the creation of additional vacancies may result in increased diffusion. To take that effect into account we used as tungsten diffusion coefficient throughout $D_{\mathrm{W}}=10^{-4} D_{\mathrm{Fe}}$.

\subsection{Temperature dependency}

The experimental results indicate a general temperature dependence of the surface enrichment under deuterium bombardment. However, the dependencies are different for the same irontungsten system exposed at PISCES A and at the high-current ion source despite using a matched deuterium ion energy [1].

Here we study the fluence resolved enrichment of an iron sample with 10 at\% tungsten exposed to $200 \mathrm{eV}$ deuterium atoms with a low-flux of $10^{15} \mathrm{D} / \mathrm{m}^{2} \mathrm{~s}$. On the left panel of Fig. 5

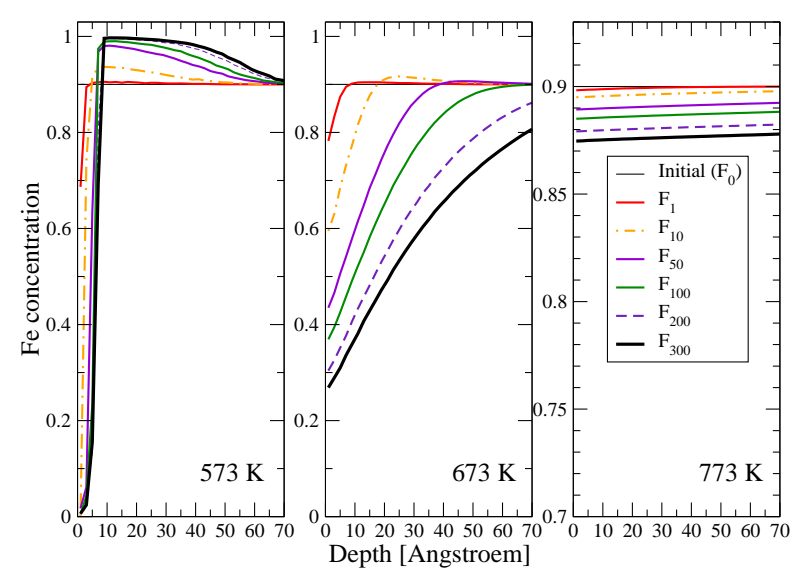

Figure 5. Comparison of the iron depth distributions as function of $200 \mathrm{eV}$ deuterium fluence for different sample temperatures of $573 \mathrm{~K}$ (left panel), $673 \mathrm{~K}$ (middle panel) and $773 \mathrm{~K}$ (right panel). The deuterium flux in the simulation was $10^{15} \mathrm{D} / \mathrm{m}^{2} \mathrm{~s}$. With raising temperature the amount of tungsten enrichment decreases, being reduced from a nearly $100 \%$ tungsten surface at $573 \mathrm{~K}$ to a few percent at $773 \mathrm{~K}$. On the other hand the extend of the iron depleted zone increases dramatically. This has to be considered in the interpretation of measured RBS data, where the resolution limit is around $10 \AA$ (see text for more details).

the iron concentrations for the sequence of fluence of 1, 10,50,100,200 and $300 \times 10^{20} \mathrm{D} / \mathrm{m}^{2}$ are displayed. With increasing fluence a very shallow layer $(\sim 0.1-0.3 \mathrm{~nm})$ at the surface becomes progressively enriched with tungsten. At the same time a pronounced increase of the sub-surface iron concentration from 90 at.\% to almost 100 at.\% due to recoil implantation is visible. This recoil peak is almost completely suppressed if the temperature is increased by $100 \mathrm{~K}$ (see middle panel of Fig. 5). At the same time the iron depleted zone has become much wider, extending into a depth of $100 \AA$, far beyond any influence of the deuterium ions 
although the surface concentration of tungsten at $673 \mathrm{~K}$ does not longer approach 100 at.\% but is limited to slightly below 80 at.\%. Finally, at temperatures of $773 \mathrm{~K}$ the preferential erosion of iron results in an enrichment of tungsten of only a few at.\%, because the high iron mobility suppresses the build-up of a significant concentration gradient.

The temperature dependent extend of the depleted zone provides a reasonable explanation of recent Rutherford backscattering data on surface enrichment of EUROFER under $200 \mathrm{eV}$ deuterium irradiation where the enrichment was surprisingly largest at intermediate temperatures [23]: The depth resolution of RBS and ERDA is typically insufficient to resolve structures below 1-3 nm even at the surface (however, see [24] for means to achieve better resolution) and thus the measured signal is in first order provided by the average of the concentration within the unresolved depth range. Computing this average value for the three temperatures displayed in Fig. 5 it is evident that the medium temperature case of $673 \mathrm{~K}$ would yield the largest surface tungsten peak in an RBS measurement, contrary to the fact that the most pronounced $\mathrm{W}$-surface enrichment happens at the lowest temperature of $573 \mathrm{~K}$.

\subsection{Flux dependency}

Initially the influence of the irradiation flux on the concentration profiles was not considered (cf. [1]) and the enrichment was discussed as function of temperature, initial composition and fluence for a given energy only. However, the steady-state concentration profiles is given by the interplay of the fluxes of particles being sputtered and the replenishment by the concentration gradient driven diffusion flux. Therefore, in a simplified description, we would expect three regimes for the different diffusive flux versus sputter flux ratios for each involved species in the material. If the diffusion flux dominates than the surface composition will remain constant under ion irradiation, because the formation of a concentration gradient by collision induced processes will be suppressed by the resulting diffusion. On the other hand, if the fluxes driven by collision cascades are dominating than the replenishment by diffusion is negligible. And then there will be a transition regime where these two contributions are of comparable magnitude and some intermediate profile will result. It should be pointed out that the required fluence until an steady-state situation is reached can be very large, because on of the fluence scales is set by the ratio of recoil implantation depth and sputter yield. If the sputter yield is low (i.e. for low-energy $\mathrm{D}$ on tungsten) this ratio can be very large.

In Fig. 6 we compare iron depth distributions as function of $200 \mathrm{eV}$ deuterium fluence for different deuterium fluxes, $10^{15} \mathrm{D} / \mathrm{m}^{2} \mathrm{~s}$ (left panel), $10^{19} \mathrm{D} / \mathrm{m}^{2} \mathrm{~s}$ (middle panel) and $10^{23} \mathrm{D} / \mathrm{m}^{2} \mathrm{~s}$ (right panel). The initial composition of the sample was 90 at.\% iron and 10 at.\% tungsten and the sample temperature in the simulation was $673 \mathrm{~K}$. For the lowest flux we see a gradually build up of a surface zone where tungsten is enriched. The influence of diffusive effects results in an iron depletion extending far beyond the deuterium ion range. The diffusion also suppresses the formation of an recoil implantation peak, remnants of which can be seen at the lowest fluences. For a deuterium fluxes of $10^{19} \mathrm{D} / \mathrm{m}^{2} \mathrm{~s}$ the simulated concentration profiles are quite different. The depleted zone shrinks to almost the ion range, indicating the 
vanishing influence of the diffusion and the surface enrichment of tungsten approaches 100 at.\%. A further increase of the ion flux by four order of magnitude yields the same steady-state profile.

Indeed, estimating the deuterium flux where the sputter flux of iron for the present parameters $\left(200 \mathrm{eV} \mathrm{D}\right.$, sputter-yield $\left.\mathrm{Y}_{\mathrm{Fe}}=0.03\right)$ matches the diffusion flux of iron in the tungsten iron-system at $673 \mathrm{~K}\left(\mathrm{D}=7.97 \times 10^{-25} \mathrm{~m}^{2} / \mathrm{s}\right)$ assuming a concentration gradient of $0.0849 \mathrm{at} / \AA^{4}$ (corresponding to a depletion within $1 \AA$ ) results in a flux of $2.3 \times 10^{16} \mathrm{D} / \mathrm{m}^{2} \mathrm{~s}$. This supports the conclusion that the transition regime is situated between the lower flux of $10^{15} \mathrm{D} / \mathrm{m}^{2} \mathrm{~s}$ and $10^{19} \mathrm{D} / \mathrm{m}^{2} \mathrm{~s}$.

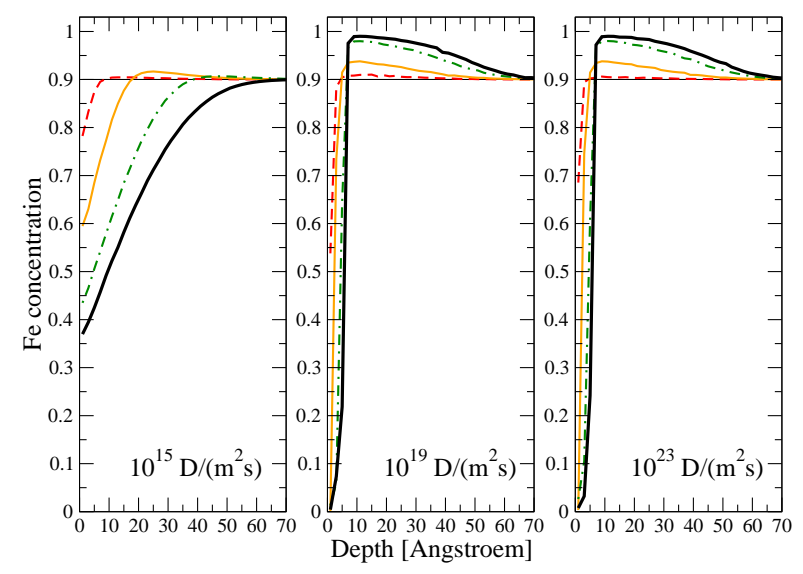

Figure 6. Comparison of the iron depth distributions as function of $200 \mathrm{eV}$ deuterium fluence for different deuterium fluxes of $10^{15} \mathrm{D} / \mathrm{m}^{2} \mathrm{~s}$ (left panel), $10^{19} \mathrm{D} / \mathrm{m}^{2} \mathrm{~s}$ (middle panel) and $10^{23} \mathrm{D} / \mathrm{m}^{2} \mathrm{~s}$ (right panel). The sample temperature in the simulation was $673 \mathrm{~K}$ and the largest deuterium fluence (corresponding to the thick solid black line) was $10^{22} \mathrm{D} / \mathrm{m}^{2}$. With increasing flux the amount of tungsten enrichment increases, reaching almost $100 \%$ tungsten surface already at a deuterium flux of $10^{19} \mathrm{D} / \mathrm{m}^{2} \mathrm{~s}$. An increase of the flux to $10^{23} \mathrm{D} / \mathrm{m}^{2} \mathrm{~s}$ does not result in further changes of the concentration profile. Please note the suppression of the iron recoil implantation peak at low fluxes.

\subsection{Concentration dependency}

The dependence of the surface enrichment of tungsten on the initial composition is of interest for several reasons: The initial composition is a robust parameter for experimental studies. This allows to test the model predictions and the physics understanding. Furthermore, the use of tungsten in reduced-activation ferritic / martensitic (RAFM) steel was no primary design target, because the process of sputtering induced surface enrichment was not considered at all. If the beneficial surface enrichment process depends sensitively on the initial tungsten concentration research attempts towards optimized initial tungsten concentrations should be considered. In the following we consider three different initial compositions of the binary iron-tungsten system with 0.5 at.\%, 1 at.\% and 10 at.\% tungsten. The iron-concentration profiles in Fig. 7 are simulated based on a sample temperature of $873 \mathrm{~K}$ and a $200 \mathrm{eV}$ deuterium flux of $10^{21} \mathrm{D} / \mathrm{m}^{2} \mathrm{~s}$. The surface concentration of tungsten is slightly above 10 at.\% 
for the sample with the lowest initial concentration, above 25 at. $\%$ for a bulk concentration of 1 at.\% tungsten and approaching 90 at.\% for a initial tungsten concentration of 10 at.\%. The surface concentration of tungsten is monotonically related to the bulk concentration. Indeed, as can be derived from Fig.8, for some conditions small variations of a low initial tungsten concentration by e.g. 1 at.\% can change the enriched surface concentration by more than 10 at.\%. It would therefore appear worthwhile to compare the enrichment properties of steels with different bulk concentrations of tungsten, e.g. EUROFER ( 0.5 at.\% tungsten) and F82H (1 at.\% tungsten) under low-energy ion bombardment.

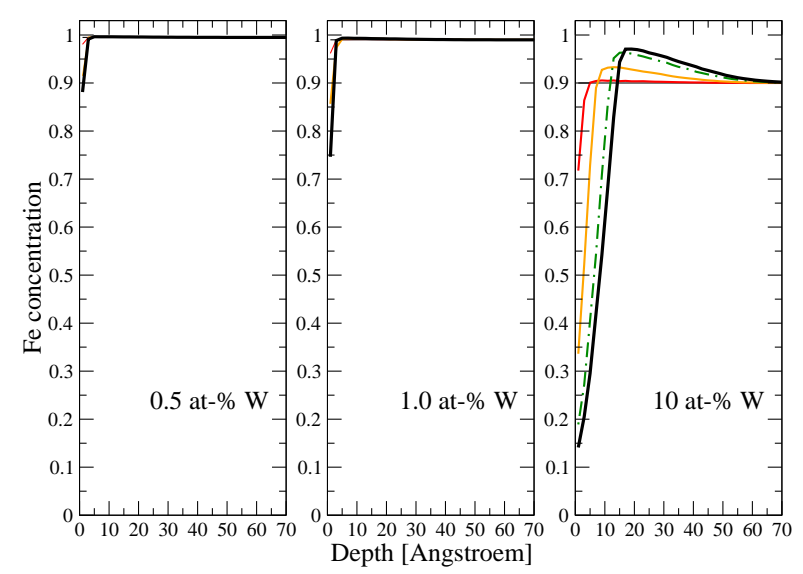

Figure 7. Comparison of the iron depth distributions as function of the initial concentration of tungsten in the sample. The sample temperature was $873 \mathrm{~K}$ and the deuterium flux of 200 $\mathrm{eV}$ deuterium ions was $10^{21} \mathrm{D} / \mathrm{m}^{2} \mathrm{~s}$. In left panel the iron concentration profile as function of fluence for an initial tungsten concentration of 0.005 is displayed. The middle and right panel provide the depth profiles for the same fluences for a tungsten concentration of 0.01 and 0.1 , respectively. With increasing bulk concentration of tungsten the surface concentration of tungsten increases. At the same time the width of the enriched zone increases. Please note: Continuous iron recoil implantation is present in all three cases, although it is only visible in the panel on the right hand side. The suppression of the iron recoil implantation peak in the left and middle panel is (unlike Fig. 5) a consequence of the use of concentration on the ordinate (with its upper limit of 1).

\section{Conclusion and Outlook}

The surface enrichment of a binary iron-tungsten model system under low-energy ion irradiation has been studied as function of fluence, flux, temperature and sample composition. For this purpose a diffusion module was added to the Monte Carlo code SDTrimSP. With this enhancement the joint effects of implantation, sputtering, material mixing and thermal diffusion can be analyzed. The experimental data of $[1,2]$ can qualitatively be explained by the interaction of preferential erosion and diffusion driven replenishment. The simulations also suggest an explanation for the surprising result that RBS measurements seem to indicate the strongest enrichment at intermediate temperatures. For the iron-tungsten model system its activation enthalpy for diffusion appear to limit an effective surface enrichment to lower 


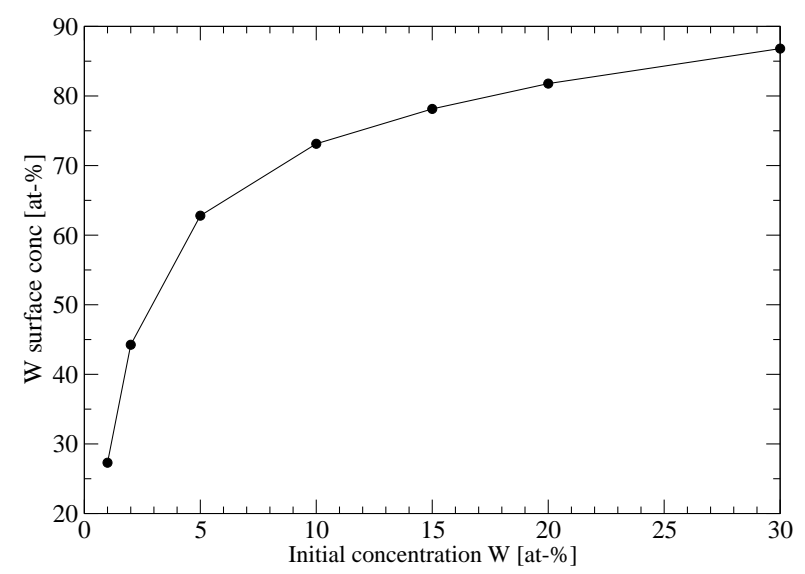

Figure 8. Tungsten surface concentration as function of the initial (bulk) sample composition. The largest sensitivity of the surface tungsten concentration on the bulk concentration is present at low bulk tungsten concentrations. Simulation parameters: sample temperature 673 $\mathrm{K}$, deuterium energy $200 \mathrm{eV}$ and a flux of $10^{15} \mathrm{D} / \mathrm{m}^{2}$ s deuterium ions.

temperatures. However, the concentration profiles are dominated by the interplay of sputterfluxes and diffusion-driven fluxes. Therefore, even at elevated temperatures a sufficiently high incident flux of low-energy deuterium results in the same enriched concentration profile as it is observed at lower temperatures. The strong dependency of the tungsten surface concentration on the initial tungsten bulk concentration observed in the simulation suggests an experimental validation of the model with steels of different tungsten content (e.g. EUROFER and F82H).

\section{Acknowledgment}

This work has been carried out within the framework of the EUROfusion Consortium and has received funding from the Euratom research and training programme 2014-2018 under grant agreement No 633053. The views and opinions expressed herein do not necessarily reflect those of the European Commission. 


\section{References}

[1] Roth J, Sugiyama K, Alimov V, Höschen T, Baldwin M and Doerner R 2014 J. Nucl. Mater. 454 1-6

[2] Sugiyama K and et al 2015 J. Nuclear Materials (in press)

[3] Möller W, Eckstein W and Biersack J 1988 Comput. Phys. Commun. 51 355-368

[4] Eckstein W, Shulga V I and Roth J 1999 Nucl. Instrum. Meth. B 153 415-421

[5] Schmid K, Roth J and Eckstein W 2001 J. Nucl. Mater. 290-293 148-152

[6] Philibert J 1991 Atom Movements - Diffusion and Mass Transport in Solids (Les Ulis Cedex: Les Editions de Physique)

[7] Mehrer H 2005 Diffusion in Condensed Matter (Berlin: Springer) chap 1. Diffusion: Introduction and Case Studies in Metals and Binary Alloys

[8] Sauer F and Freise V 1962 Z. Elektrochem. 66353

[9] Smigelskas A and Kirkendall E 1947 Trans. AIME 171 130-142

[10] Manning J 1967 Acta Metall. 15817

[11] Press W, Teukolsky S, Vetterling W and Flannery B 2007 Numerical Recipes 3rd ed (Cambridge: Cambridge University Press)

[12] Ames W 1992 Numerical Methods for Partial Differential Equations 3rd ed (New York: Academic Press)

[13] Crank J 1975 The Mathematics of Diffusion (Oxford: Clarendon)

[14] Smith G 1978 Numerical Solution of Partial Differential Equations: Finite Difference Methods (Oxford: Oxford University Press)

[15] Solin P 2005 Partial Differential Equations and the Finite Element Method (New Jersey: John Wiley)

[16] Eckstein W 2000 Nucl. Instrum. Meth. B 171 435-442

[17] Zuhr R A, Roth J, Eckstein W, von Toussaint U and Luthin J 2001 J. Nucl. Mater. 290-293 162-165

[18] Ziegler J F, Biersack J P and Littmark U 1985 The Stopping and Range of Ions in Solids (New York: Pergamon Press) see also: http://www.srim.org/

[19] Perez R and Torres D 2011 Appl. Phys. A 104 329-333

[20] Alberry P and Haworth C 1974 Metal Science 8 407-412

[21] Takemoto S, Nitta H, Iijima Y and Yamazaki Y 2007 Philosophical Magazine 87 1619-1629

[22] Kupalova I and Zemskii S 1968 Metallovedenie i Termicheskaya Obrabotka Metallov 2 10-12

[23] Sugiyama K, Roth J, Doerner R, Balden M, von Toussaint U and Jacob W 2015 Sputtering behaviour of eurofer steel by energetic deuteron bombardment at various temperatures Conference on Plasma Facing Materials and Components, Poster P69

[24] Neumaier P, Dollinger G, Bergmaier A, Genchev I, Görgens L, Fischer R, Ronning C and Hofsäss H 2001 Nuclear Instruments and Methods in Physics Research Section B: Beam Interactions with Materials and Atoms 18348 - 61 ISSN 0168-583X ion Beam Techniques with Atomic Layer Resolution URL http://www.sciencedirect.com/science/article/pii/S0168583X01003470 